\title{
Conjunctival Limbal Autograft Combined with Amniotic Membrane Transplantation to Treat a Moderate Chemical Eye Injury
}

\author{
Cem Cankaya
}

Department of Ophthalmology, Inonu University Faculty of Medicine, Malatya, Turkey

\begin{abstract}
A 25-year-old male patient presented at the clinic with chemical trauma to the left eye. His visual acuity according to Snellen chart measurement was $0.05 / 10$ in the left eye and $10 / 10$ in the right eye. On slit lamp examination, conjunctival hyperemia and limbal ischemia were observed between the I o'clock and 6 o'clock hours (approximately 5 clock hours). The cornea was edematous in this area, and a corneal epithelial defect adjacent to the limbal ischemia area was present. The anterior chamber, iris, and lens were normal; the fundus could not be concurrently evaluated. Conjunctivalization and excessive neovascularization were observed in the limbal ischemic area at the end of the fourth week of medical treatment. Visual acuity in the left eye was 0.3 and the epithelial defect had healed. At the end of the sixth week, a conjunctival limbal autograft (CLAU) combined with amniotic membrane (AM) transplantation surgery was performed. Subsequently, the corneal neovascularization was noted to have completely vanished in the ischemic area where the CLAU and AM transplantation were performed. Visual acuity improved to 0.7 and the symptoms of the patient decreased.
\end{abstract}

Keywords: Amniotic membrane transplantation, chemical eye injury, conjunctival limbal autograft

\section{Introduction}

Chemical and thermal corneal burns can cause severe anterior segment complications leading to rapid and progressive destruction. Chemical burns account for $12 \%$ of all ocular traumas. The severity of these complications is related to the concentration of the offending agent, the $\mathrm{pH}$, the duration of exposure, and the damage to the ocular surface and tissues (I).

Ocular chemical burns are the most common cause of limbal stem cell deficiency, and are characterized by corneal epithelial defects, stromal inflammation, neovascularization, conjunctivalization, and corneal opacification (2). In the treatment of limbal deficiency and conjunctivalization, conjunctival limbal autograft (CLAU) combined with AM transplantation surgery is a good option in the event that CLAU transplantation alone is not sufficient to cover the ischemic limbal area.

\section{Case Report}

A 25 -year-old male patient presented at the clinic with a work-related chemical trauma to the left eye. According to the patient, the substance he was exposed to was lime. Ocular discomfort, foreign body sensation, burning sensation, blurred vision, and excess tearing in the left eye were reported. Snellen chart visual acuity was measured at $0.05 / 10$ in the left eye and $10 / 10$ in the right eye. Mild upper eyelid edema as well as several pieces of lime embedded in conjunctival epithelium under the upper eyelid were observed during slit lamp examination. The conjunctiva was hyperemic and there was limbal ischemia between the I o'clock and 6 o'clock hours. The cornea was edematous in this area, and a corneal epithelial defect adjacent to limbal ischemic area was observed. The anterior chamber, iris, 
Table I. Roper-Hall classification for the severity of ocular surface burns

\begin{tabular}{llll} 
Grade & \multicolumn{2}{c}{ Clinical findings } & Prognosis \\
\cline { 2 - 3 } & Cornea & Conjunctiva/limbus & \\
\hline I. & Corneal epithelial damage & No limbal ischemia & Good \\
II. & Corneal haze, iris details visible & $<I / 3$ limbal ischemia & Good \\
III. & Total epithelial loss, stromal haze, & $1 / 3-I / 2$ limbal ischemia & Guarded \\
IV. & and iris details obscured & & \\
& Cornea opaque, iris and & $>I / 2$ limbal ischemia & Poor \\
\hline
\end{tabular}

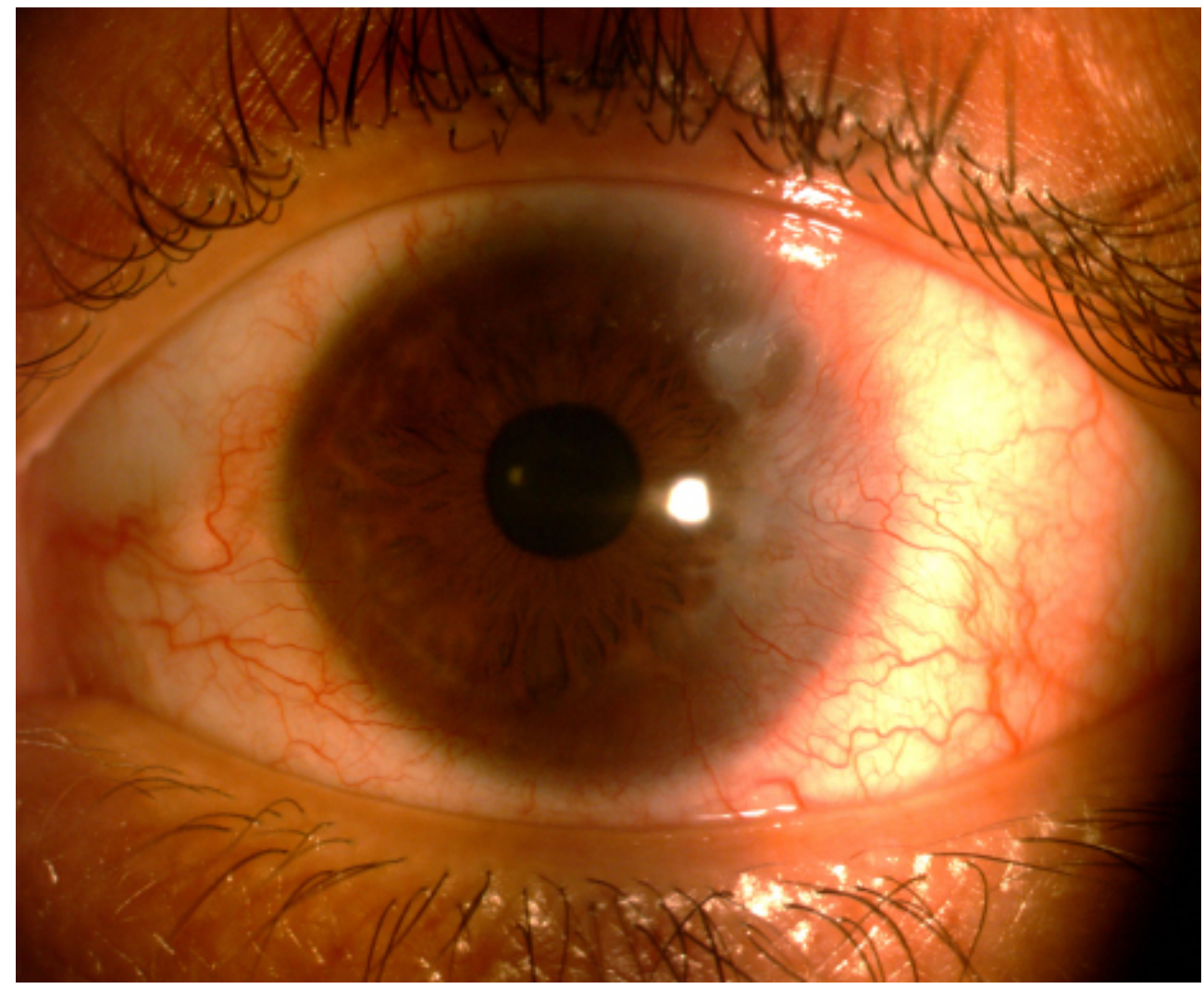

Figure I. Conjunctivalization and excessive diffuse superficial neovascularization can be seen between the I o'clock and 6 o'clock hours due to limbal deficiency.

and lens were normal; the fundus could not be concurrently evaluated. Intraocular pressure and ocular motility were normal. According to the Roper-Hall classification (Table I), the evaluation was a grade II ocular surface burn. The right eye was uninjured. The patient was hospitalized. The eyes and surrounding tissues were irrigated with physiological saline for 30 minutes.

Topical antibiotic drops (moxifloxacin, Vigamox; Alcon, Ft. Worth, TX, USA) were administered to prevent infection, as well as $1 \%$ prednisolone acetate at the first week and after the 3rd week to allow for epithelial recovery during the second week, tetracycline ointment, cyclopen- tolate drops, preservative-free artificial tears, and oral vitamin $C$ ( 2 g daily). Conjunctivalization and excessive diffuse superficial neovascularization were observed in the limbal ischemic area at the end of the fourth week of medical treatment (progression of the conjunctival epithelium on the cornea, loss of limbal palisades of Vogt and peripheral superficial neovascularization findings alone or in combination with other findings were evaluated as limbal dysfunction) (Fig. I). Visual acuity was assessed as 0.3 and the epithelial defect improved. Topical bevacizumab $(5 \mathrm{mg} / \mathrm{mL})$ was added to the treatment for 2 weeks 4 times a day. At the end of the sixth week, conjunctival limbal autograft 


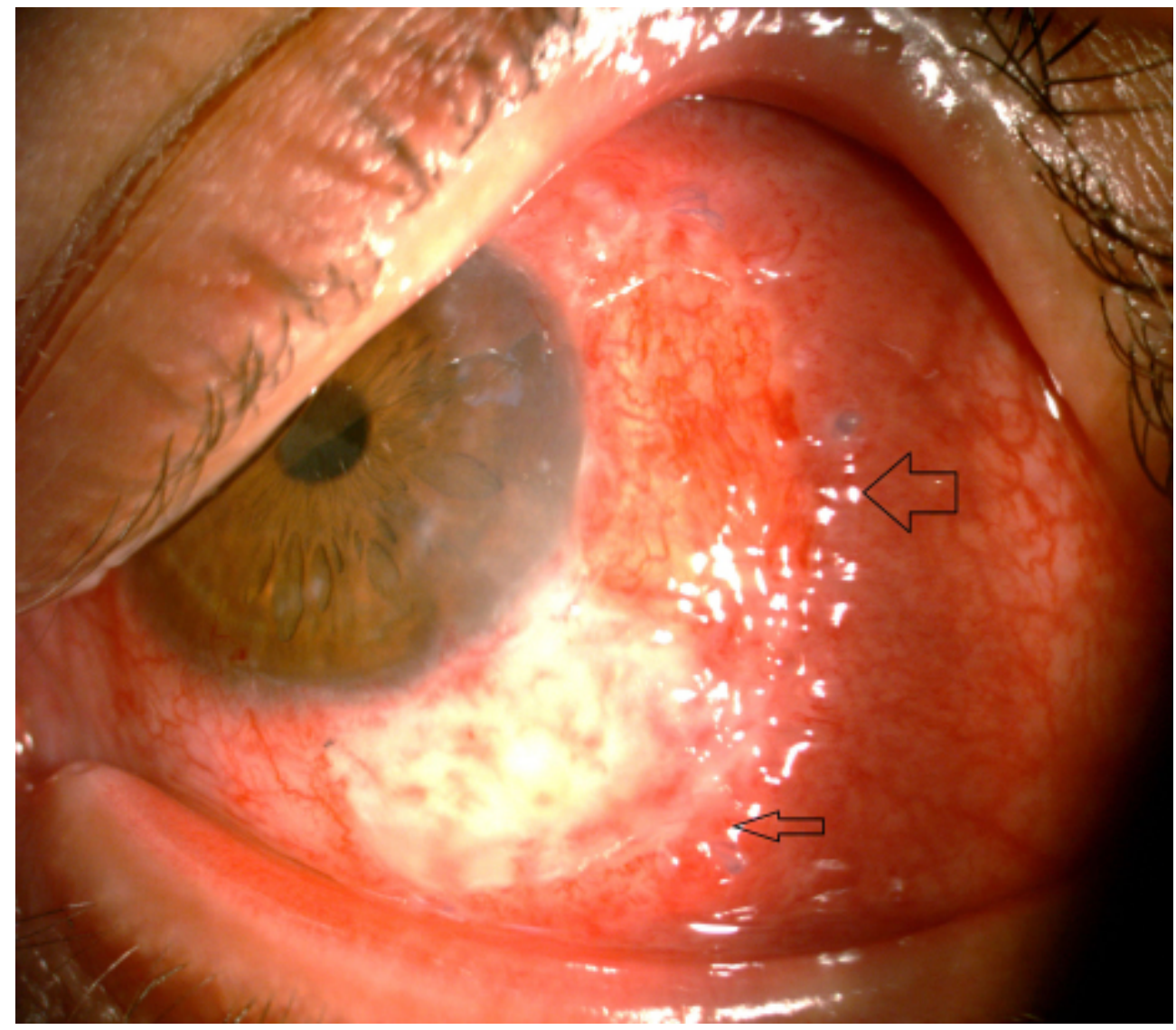

Figure 2. The thick arrows indicate the conjunctival limbal autograft, and the thin arrows designate the amniotic membrane.

(CLAU) surgery combined with amniotic membrane (AM) transplantation was performed (Fig. 2).

Following the administration of sub-Tenon anesthesia with $2 \%$ lidocaine, a conjunctival peritomy and superficial keratectomy, including the limbal area, were performed to remove the vascularized pannus tissue. Adequate hemostasis was provided with cautery. A CLAU consisting of a limbal arc of $6 \mathrm{~mm}$ in length (i.e., 2 clock hours or $60^{\circ}$ ), with I mm of peripheral cornea and $8 \mathrm{~mm}$ of the conjunctiva was removed from the superior limbus of the healthy eye. At this stage, we were careful not to remove Tenon's capsule or episcleral tissue. The graft tissue was sutured to the quadrant with the most limbal deficiency in the recipient bed, in accordance with the anatomical position. AM transplantation was performed to the bare area approximating the I o'clock to 2 o'clock-hour quadrant. The amniotic membrane was placed such that the stromal side would be in contact with the ocular surface and cover the excision zone. After the AM was fixed with episcleral sutures 2 to $3 \mathrm{~mm}$ behind the limbus, it was sutured at the conjunctival margins around the limbus with $8 / 0$ monofilament silk sutures. On the limbal side, 10/0 nylon sutures were used. In the postoperative period, the patient was treated with moxifloxacin drops (Vigamox; Al- con, Ft. Worth, TX, USA) 4xI, artificial tears (hydroxypropyl methyl cellulose, Tears Naturale Free, Alcon, Ft. Worth, TX, USA) $6 \mathrm{xI}$, and prednisolone acetate drops (Pred Forte; Allergan plc, Dublin, Ireland) $4 \times 1$. Corneal neovascularization was seen to have completely receded in the ischemic area where the combined CLAU and AM transplantation was performed at subsequent visits (Fig. 3). Visual acuity improved to 0.7 and the symptoms of the patient decreased.

\section{Discussion}

Chemical (alkali and acid) injury of the conjunctiva and cornea is a true ocular emergency and requires immediate intervention. Chemical injuries to the eye can produce extensive damage to the ocular surface and anterior segment, leading to visual impairment and disfigurement. Early recognition and treatment ensures the best possible outcome for this potentially blinding condition (3).

Ocular chemical burns are the most common cause of limbal stem cell deficiency, and are characterized by chronic epithelial defects, stromal inflammation, neovascularization, conjunctivalization, and corneal opacification.

In the management of chemical burns, removal of the agent from the ocular surface and inflammation suppression 


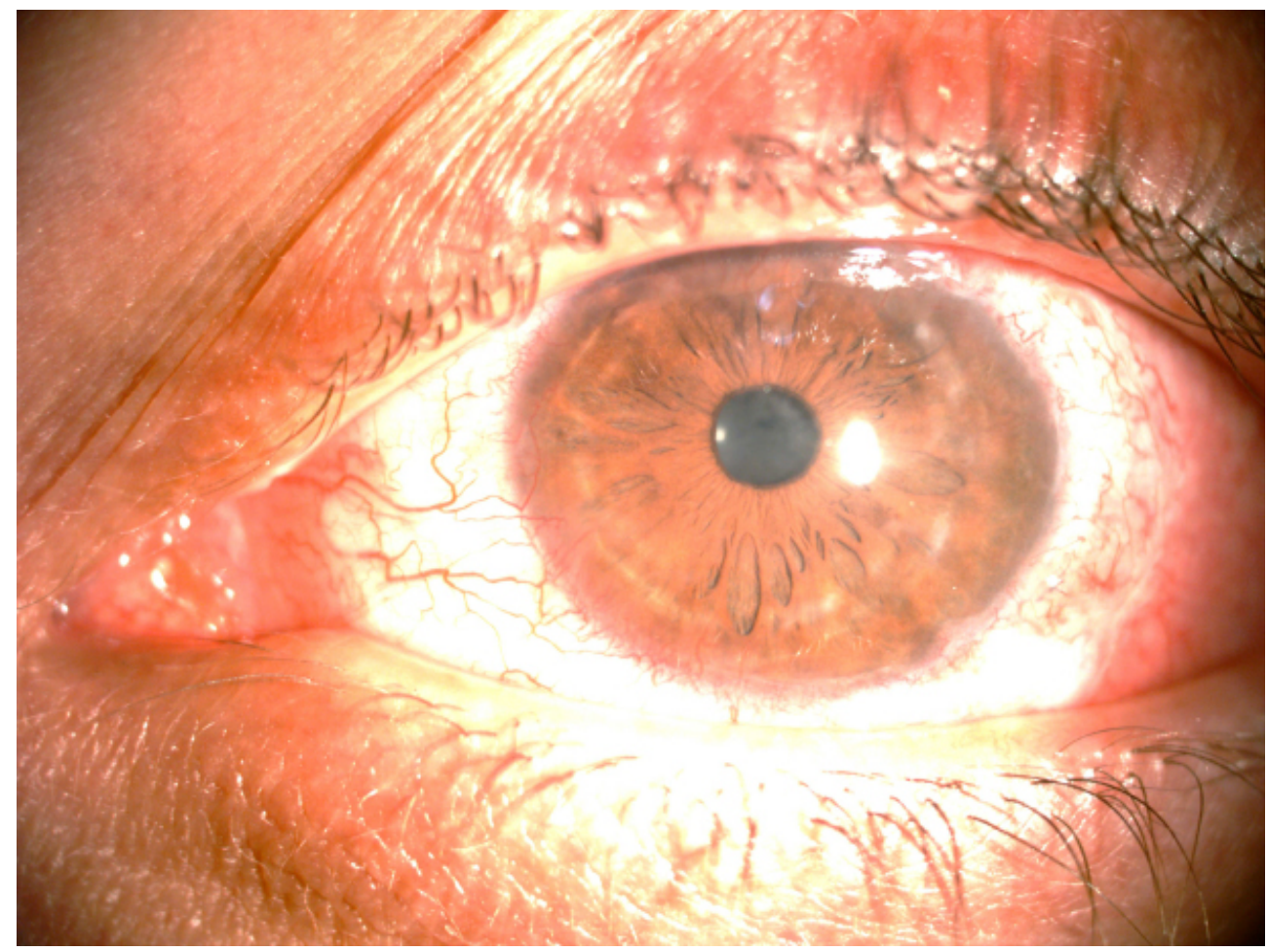

Figure 3. An image illustrating the ocular surface of the patient 4 weeks after surgery.

is essential in the acute phase; however, management of complications and providing a healthy ocular surface is important in the chronic period (4). In recent years, satisfactory results have been obtained in chemical eye injuries by combining appropriate medical treatment in the acute period with ocular surface reconstruction (5). Appropriate medical treatment was applied in the acute phase in our case. However, neovascularization and conjunctivalization developed in the chronic period due to the development of limbal deficiency secondary to the chemical injury. This was treated with a CLAU of limited size obtained from the healthy eye combined with AM transplantation for the bare area in order to obtain a smooth ocular surface.

AM transplantation performed during the acute or chronic period in the treatment of corneal burns helps to protect the conjunctival surface and reduce limbal stromal inflammation. Furthermore, AM transplantation performed to improve the epithelial defects also provides pain control in ocular surface reconstruction $(5,6)$. AM transplantation also has the advantages of reducing perilimbal inflammation, creating healthy corneal epithelium, reducing corneal neovascularization, and increasing the success of future limbal stem cell transplantation and/or penetrating keratoplasty in ocular surface reconstruction (7).

Meller et al. (8) reported that I 3 eyes of II patients who underwent $A M$ transplantation after conventional medical treatment provided epithelialization, and only those with grade IV disease developed limbal stem cell failure. Tejwani et al. (9) reported that $92.9 \%$ of the patients who were followed up for chemical corneal burn had epithelial recovery, $84.6 \%$ of them had improvement in symptoms, and $63.5 \%$ of them had success in ocular surface reconstruction with AM transplantation. We performed AM transplantation during the chronic period in our case. Our case had a grade II corneal burn according to the Roper-Hall classification, and the epithelial defect was limited to the limbal ischemic area. As a result of the improvement of the epithelial defect with medical treatment, it was not necessary to perform AM transplantation in the acute phase.

Satisfactory results can be obtained with CLAU in patients with a unilateral limbal stem cell deficiency. However, the quantity of CLAU to be removed from the healthy eye is limited. Conjunctival limbal stem cell transplantation poses a potential risk of causing limbal stem cell deficiency in healthy eyes. Therefore, although the overall risk is low if the donor eye is truly healthy (i.e., without long-term contact lens wear or subclinical exposure to original trauma) and if $<6$ clock hours of limbal tissue is removed, the risk is minimized (I0). Therefore, we removed a limited amount of CLAU to avoid any limbal deficiency in healthy eye. The amount of CLAU was not sufficient to cover the entire limbal ischemia-vascularization zone, and the AM was applied to the bare area in the I o'clock to 2 o'clock area.

In conclusion, chemical burns can cause damage to the eye on a broad spectrum, from mild to severe. Healing, stabilization, and management of complications on the ocular 
surface are important in patient follow-up. CLAU is a good surgical alternative in corneal vascularization and ocular surface disorders, in particular in cases of limbal ischemia or deficiency in only I eye. However, in patients with extensive vascularization, if the limited CLAU obtained to avoid limbal deficiency in the healthy eye is not sufficient to cover the ischemic area, CLAU combined with AM transplantation can be a good surgical alternative.

\section{Disclosures}

Informed consent: Written informed consent was obtained from the patient for the publication of the case report and the accompanying images.

Peer-review: Externally peer-reviewed.

Conflict of Interest: None declared.

\section{References}

I. Tuft SJ, Shortt AJ. Surgical rehabilitation following severe ocular burns. Eye 2009;23:1966-7I. [CrossRef]

2. Fish R, Davidson RS. Management of ocular thermal and chemical injuries, including amniotic membrane therapy. Curr Opin Ophthalmol 2010;21:317-21. [CrossRef]

3. Pargament JM, Armenia J, Nerad JA. Physical and chemical injuries to eyes and eyelids. Clin Dermatol 2015;33:234-7. [CrossRef]

4. Tsubota K, Toda I, Saito H, Shinozaki N, Shimazaki J. Recon- struction of the corneal epithelium by limbal allograft transplantation for severe ocular surface disorders. Ophthalmology 1995; 102: 1486-96. [CrossRef]

5. Shimazaki J, Yang HY, Tsubota K. Amniotic membrane transplantation for ocular surface reconstruction in patients with chemical and thermal burns. Ophthalmology 1997; 104:2068-76. [CrossRef]

6. Gomes JA, Santos MS, Ventura AS, Donato WB, Cunha MC, Höfling-Lima AL. Amniotic membrane with living related corneal limbal/conjunctival allograft for ocular surface reconstruction in Stevens-Johnson syndrome. Arch Ophthalmol 2003; | 2 |:|369-74. [CrossRef]

7. Tseng SC, Prabhasawat P, Barton K, Gray T, Meller D. Amniotic membrane transplantation with or without limbal allografts for corneal surface reconstruction in patients with limbal stem cell deficiency. Arch Ophthalmol 1998; I 16:43 I-4I. [CrossRef]

8. Meller D, Pires RT, Mack RJ, Figueiredo F, Heiligenhaus A, Park WC, et al. Amniotic membrane transplantation for acute chemical or thermal burns. Ophthalmology 2000;107:980-9. [CrossRef]

9. Tejwani S, Kolari RS, Sangwan VS, Rao GN. Role of amniotic membrane graft for ocular chemical and thermal injuries. Cornea 2007;26:2I-6. [CrossRef]

10. Kheirkhah A, Raju VK, Tseng SC. Minimal conjunctival limbal autograft for total limbal stem cell deficiency. Cornea 2008;27:730-3. [CrossRef] 